\title{
A Double-Blinded, Prospective Trial with A New Formula in Distressed and Regurgitating Infants
}

\author{
Y. Vandenplas*, T. Devreker and B. Hauser
}

Universitair Ziekenhuis Brussel Kinderen, Brussels, Belgium

\begin{abstract}
Aim: Infant regurgitation and distress are common but are a major cause of parental anxiousness. The efficacy of dietary management is contradictory.

Material and Methods: Twelve exclusively formula fed infants crying for more than 3 hours/day since at least 3 weeks and regurgitating several times after each feeding were enrolled in a double blinded cross-over intervention trial with two innovative formulas ("G1" and "G3"), both developed by Novalac. Prior to inclusion, all infants had been put on at least three different commercialized AR-formulae and/or formulae for digestive comfort and at least one extensive hydrolysate without improvement. All infants had been treated unsuccessfully with prokinetics (domperidone or cisapride) and acidblocking drug medication $\left(\mathrm{H}^{2}\right.$ receptor antagonists and/or generic omeprazole and/or alginates).
\end{abstract}

Results: One formula ("G3") scored significantly better than the other, resulting in a significant decrease in regurgitation and crying, an overall subjective well-being amelioration of the baby and enhanced gastric emptying. All 12 mothers preferred the same test formula, despite the cross-over and blinded design. Stool patterns and aspect with "G3" was comparable to defecation in breast fed infants.

Conclusion: Although the number of infants is limited, one of the test formulas, G3, combining a specific partial whey hydrolysate with a combination of thickeners (bean gum and specially processed tapioca starch) was a) effective in alleviating the symptoms where other thickened and extensively hydrolyzed formulas as well as prokinetics and acid blocking drugs had failed and b) more effective than the other tested formula combining 2 thickeners (locust bean and starch) widely used in anti-regurgitation formulas, G1.

\section{INTRODUCTION}

Gastro-oesophageal reflux (GOR), defined as the passage of gastric contents into the oesophagus, is a normal physiologic process. Symptoms of GOR include regurgitation and vomiting. Most episodes of reflux are into the distal oesophagus, are brief, and asymptomatic. Regurgitation is defined as passage of refluxed gastric contents into the oral pharynx. Vomiting is a coordinated reflex that results in the expulsion of the refluxed gastric contents from the mouth. GOR-disease (GORD) occurs when gastric contents refluxes into the oesophagus or oropharynx and produces troublesome symptoms.

Recurrent regurgitation occurs in 50 to $70 \%$ of infants in the first three to four months of life, but resolves spontaneously in nearly all of these infants [1-3]. Although the incidence of regurgitation is not different in formula and breast fed infants [1], the phenomenon is perceived different by the parents. This is likely to be related to the motivation to choose for breast or formula feeding. In Belgium, mothers breastfeed because of the positive effects of mother's milk on the health of the baby, more specifically on the development of the immune system. Mothers choose for formula because of convenience (data from unpublished questionnaire in Belgium). Parents do not usually perceive vomiting

*Address correspondence to this author at the UZ Brussel Kinderen, Laarbeeklaan 101, 1090 Brussels, Belgium; Tel: + 32247757 80; Fax: + 322 47757 83; E-mail: yvan.vandenplas@uzbrussel.be as a problem when it occurs not more often than once daily, but they are more likely to be concerned when vomiting is more frequent, or when the infant cries frequently or with vomiting. About $20-25 \%$ of the parents of formula fed babies seek medical help because of frequent regurgitation and inconsolable crying in formula-fed infants.

Evidence supporting the theory that reflux causes esophageal pain and hence irritability or sleep disturbance in infancy is largely extrapolated from studies in adults [4,5]. Using simultaneous video and esophageal $\mathrm{pH}$ monitoring, an association between grimacing and reflux episodes was demonstrated [6]. Feeding problems occur in up to $30 \%$ of infants younger than 1 month undergoing endoscopy, with a diagnosis of oesophagitis in $21 \%$ of them [7].

The primary goal of therapy is to relieve the patient's symptoms and to improve the overall subjective well-being of the baby and the parent(s). We aimed in this trial to test the efficacy of two new formulas on infant regurgitation and distress.

\section{MATERIAL AND METHODS}

Twelve consecutive, well thriving exclusively formula fed infants but crying for more than 3 hours per day since at least 3 weeks and regurgitating several times after each feeding were included in this prospective cross-over trial. According to the care-giver and the physician, there had to be a probable time-relation between feeding and infant distress. The infants were referred to a tertiary care centre because 
previous dietary and medical interventions had been unsuccessful. Prior to inclusion, all infants had been put on at least three different commercialized AR-formulae and/or formulae for digestive comfort and at least one extensive hydrolysate without improvement. All infants had been treated unsuccessfully with prokinetics (domperidone or cisapride) and acid-blocking drug medication $\left(\mathrm{H}^{2}\right.$ receptor antagonists and/or generic omeprazole and/or alginates).

Two formulas were developed for clinical evaluation (composition: Table 1). "G1" is a $80 / 20$ casein/lactalbumin formula thickened with bean gum and starch. "G3"(Novalac AR Digest) is a partial whey hydrolysate, thickened with a mixture of bean gum and tapioca starch. The working hypothesis for "G1" was that a thickened casein predominant formula may decrease regurgitation and GOR, and therefore decrease crying. The working hypothesis for "G3" was that the protein in a protein hydrolysate has improved digestibility, that gastric emptying will be enhanced, and that the thickening will also decrease regurgitation and reflux. All infants were included in a prospective, randomized, doubleblinded (parents and physicians), cross-over trial (6 infants started on G1 ( 2 weeks) and were then switched to G3 (2 weeks); 6 infants started on G3 (2 weeks) and were switched to G1 ( 2 weeks). The primary allocation (first G1 or first G3) was done according to an envelope drawing system. The medical team knew which infant was on which formula but was blinded for the composition of G1 and G3. Although the intervention period for each formula was set at 2 weeks, it was possible for a care-giver to request the change after 5 days if the improvement was estimated to be insufficient.
The care-giver could obtain the preferred formula for a longer period (up to the age of 6 months) in case of good clinical response.

As primary endpoint, parents were asked to indicate the formula of their preference. Parents were also asked to fill in a diary during the entire intervention, recording the number of episodes of regurgitation per day, hours of crying, number of feedings per day, duration and volume of each feeding, number and aspect of stools per day. Finally, "an overall subjective well-being amelioration of the baby as perceived by the parents (well-being)" was asked for the comfort of the baby and of the parents (Evaluation through a scale from $0=$ Excellent to 5 =Worst).

Three gastric emptying studies with a 13-C acetate breath test were performed: one at baseline with a commercialized starch thickened formula, one at the end of the period during which the first test-formula given, and one at the end of the second intervention period with the second test-formula. The intake was "at libitum". Since all infants were thriving well, other investigations were not performed.

The results were statistically analyzed using the Friedman test. A $p$-value of 0.05 or less was accepted as statistical significance. The study protocol was approved by the local ethical committee.

\section{RESULTS}

All twelve mothers clearly preferred the "G3-test formula" and continued on this formula after the intervention period. Only 3/12 infants remained for 2 weeks on "G1".

Table 1. Composition of the Two Test-Formulae $(\mathrm{g} / 100 \mathrm{ml})$

\begin{tabular}{|c|c|c|}
\hline & G1 & G3 \\
\hline \hline Protein & 1.6 & Partial whey hydrolysate \\
\hline Whey/Casein & $80 / 20$ & 6.9 \\
\hline Carbohydrate & 7.4 & 3.7 \\
\hline Starch (\%) & 4.2 & 56.6 \\
\hline Maltodextrin (\%) & 20 & 0.4 \\
\hline Fiber & 0.2 & 3.3 \\
\hline Lipid & 3.1 & Bean gum Tapioca starch \\
\hline Thickening agent & Bean gum Starch & 63.3 \\
\hline Energy (kcal) & 63.9 & \\
\hline
\end{tabular}

Table 2. Clinical Characteristics During the Intervention Period with Each Formula (mean \pm 1 Standard Deviation)

\begin{tabular}{|c|c|c|c|}
\hline & G1 & G3 & P \\
\hline \hline Episodes regurgitation & $5.1 \pm 1.2$ & $1.8 \pm 1.2$ & 0.002 \\
\hline Crying time (min) & $84.5 \pm 50.1$ & $26.7 \pm 18.1$ & 0.003 \\
\hline Overall subjective well-being* & $4.2 \pm 1.8$ & $2.1 \pm 0.7$ & 0.005 \\
\hline Gastric emptying & $104.5 \pm 15.5 \mathrm{~min}$ & $79.2 \pm 14.0 \mathrm{~min}$ & $<0.001$ \\
\hline
\end{tabular}

Legend: Number of patients: 12 ; data are mean \pm 1 standard deviation.

Gastric emptying (13C acetate breath test) was $117.1 \pm 18.3 \mathrm{~min}$ with standard AR formula at baseline.

$*$ Evaluated through a scale from $0=$ excellent to $5=$ worse 
There were no differences in the number and volume of feedings. Stools did not differ in number or aspect. The mean number of episodes of regurgitation per day was significantly lower with "G3" (Table 2). Also crying time per day was significantly decreased with "G3". The overall subjective well-being amelioration as perceived by the parents was significantly better with "G3" than with "G1". Gastric emptying was significantly more rapid with "G3" compared to G1 and the commercialized starch thickened AR -formula.

\section{DISCUSSION}

The primary endpoint of this intervention trial was subjective since parents had to indicate their formula of preference. However, the cross-over design with 6 infants starting with "G1" and 6 infants staring with "G3" minimized the risk for bias since the composition of the formulae was only known to the company. All 12 mothers indicated "G3" as their formula of preference. All infants had been given previously at least three formulas without success; all 12 infants continued on "G3" up to the age of 6 months (maximal duration). "G3" scored significantly better than "G1": a decreased number of episodes of regurgitations, decreased crying, the overall subjective well-being amelioration of the baby and enhanced gastric emptying in 12 infants in whom previous dietary and pharmaceutical management had failed.

Knowledge on the natural history of regurgitation and vomiting is limited. The frequency of regurgitation varies largely in relation to age. Martin et al. showed that about 40 $\%$ of all 3-4 month old infants regurgitate, whereas at 12 months of age, only $5 \%$ of infants regurgitate [1]. According to an Italian observational study involving general pediatricians following 2879 infants to the age of 6 months, regurgitation was reported in almost one infant in four [8]. Formula was changed in $60 \%$ of all infants [8]. A prospective followup of 63 regurgitating infants reported a remission in all before the age of one year. However, even thereafter, feeding refusal, duration of a meal and parental feeding related distress were significantly prolonged or increased in the group with regurgitation if compared to a control group [2,3]. This observation suggests a decreased overall subjective wellbeing of the regurgitating baby and their parents, even if regurgitation has disappeared. Infants spitting during 90 days or more during their first 2 years of life are at increased risk to develop GOR symptoms at 9 years of age [1]. This may point to the fact that some of these infants spitting up frequently may have undiagnosed GOR-disease [2,3]. Conversely, a history of GOR is the most frequent underlying cause of feeding disorders in young children [3].

The infants described in this study are infants that regurgitate and cry a lot without clear evidence for the etiology of these phenomena. At least according to the interpretation of the parents, there seemed to be a causal relation between the crying and feeding. However, this is hard to confirm since the infants cry for several hours during the day and are fed about every four hours. In other words: feeding is almost because of its frequency obligatory time-related to the crying.

None of the infants included in this trial seemed to have difficulties in passing (hard) stools (another frequent problem according to the Italian observational [8]). Neither G1 nor G3 influenced the aspect and frequency of defaecation, although bean gum is said to cause more frequently watery stools [9]. As a consequence, the defecation pattern of the infants on "G3" was closer to the stool pattern of breast fed infants than with regular formula or "G1".

One of the test formulas was consequently indicated by the mothers as "the best" for their infant in a group of babies in who anti-acid medication, prokinetics, extensive hydrolysates and other formula changes had been unsuccessful. The enhanced gastric emptying may have contributed to the success of "G3", together with the increased digestibility of the proteins (partial whey hydrolysate) and the unique mixture of tapioca starch and bean gum as thickening agent. The bean gum thickens the formula in the bottle, and thus reduces air swallowing. The tapioca starch exerts its thickening effect mainly in the stomach.

The cross-over design with two test formulas minimizes the impact of spontaneous evolution.

\section{REFERENCES}

[1] Martin AJ, Pratt N, Kennedy JD, et al. Natural history and familial relationships of infant spilling to 9 years of age. Pediatrics 2002; 109: 1061-7.

[2] Nelson SP, Chen EH, Syniar GM, Christoffel KK. Prevalence of symptoms of gastroesophageal reflux during infancy. A pediatric practice-based survey. Pediatric Practice Research Group. Arch Pediatr Adolesc Med 1997; 151: 569-72.

[3] Nelson SP, Chen EH, Syniar GM, Christoffel KK. One-year followup of symptoms of gastroesophageal reflux during infancy. Pediatric Practice Research Group. Pediatrics 1998; 102(6): E67.

[4] Katzka DA, Paoletti V, Leite L, Castell DO. Prolonged ambulatory $\mathrm{pH}$ monitoring in patients with persistent gastroesophageal reflux disease symptoms: testing while on therapy identifies the need for more aggressive anti-reflux therapy. Am J Gastroenterol 1996; 91: 2110-3.

[5] Singh H, Gill PJ, Soni RK, Raizada N. Sleep pattern and night awakening in healthy infants. Ind Pediatr 1992; 29: 1373-7.

[6] Feranchak AP, Orenstein SR, Cohn JF. Behaviors associated with onset of gastroesophageal reflux episodes in infants. Prospective study using split-screen video and $\mathrm{pH}$ probe. Clin Pediatr (Phila) 1994; 33: 654-62.

[7] de Boissieu D, Dupont C, Barbet JP, Bargaoui K, Badoual J. Distinct features of upper gastrointestinal endoscopy in the newborn. J Pediatr Gastroenterol Nutr 1994; 18: 334-8.

[8] Iacono G, Merolla R, D'Amico D, et al. Paediatric Study Group on Gastrointestinal Symptoms in Infancy. Gastrointestinal symptoms in infancy: a population-based prospective study. Dig Liver Dis 2005; 37: 432-8.

[9] Vandenplas Y, Salvatore S, Hauser B. The diagnosis and management of gastro-oesophageal reflux in infants. Early Hum Dev 2005; 81: 1011-24.

(C) Vandenplas et al.; Licensee Bentham Open.

This is an open access article licensed under the terms of the Creative Commons Attribution Non-Commercial License (http://creativecommons.org/licenses/by-nc/3.0/) which permits unrestricted, non-commercial use, distribution and reproduction in any medium, provided the work is properly cited. 